\title{
Peak Average Power Ratio Reduction Technique for Better Performance with Multi Input Multi Output
}

\author{
K.Aruna Kumari, K.Sri Rama Krishna
}

\begin{abstract}
In the transmitted signal, the high peak-toaverage power ratio (PAPR) is the real disadvantage of multiple input, multiple output (MIMO) orthogonal frequency division multiplexing (OFDM) systems. Among different PAPR reduction techniques, selected mapping (SLM) is a famous strategy that accomplishes good PAPR reduction performance without signal distortion. Likewise, Partial transmit sequence (PTS) is additionally solitary of the successful techniques to decrease the PAPR in OFDM. Though, result an optimal segment issue in PTS system is measured to exist a critical concern. To progress the existing PAPR reduction techniques, we have incorporated ideal SLM and PTS based PAPR reduction strategy in parallel. By utilizing, the OGWO algorithm; the transmit succession was chosen with least PAPR above all communication antennas. The proposed PAPR reduction approach is applied independently on each transmitted antenna, and so the PAPR can be extremely reduced. Moreover, the OGWO optimization based PAPR reduction technique will provide better performance and it was been promoted as an uncomplicated way for PAPR reduction. The proposed approach will be analyzed with various novel $P A P R$ reduction schemes to show the effectiveness.
\end{abstract}

Key words - MIMO-OFDM, PAPR reduction, SLM, PTS, GWO based Opposition.

\section{INTRODUCTION}

Orthogonal frequency division multiplexing is regularly utilized for soaring information rate wireless interchanges because of its natural fault strength in a environment. [1]. Combination of MIMO system with OFDM has a major consideration for the subsequently making broadband utilization suitable to their probable of provide soaring. MIMO-OFDM have be set up in different remote correspondence values, Remote systems, overall interoperability for microwave), and Long Term Evolution [2].Power efficiency can be improve by maximizing spatial diversity. By changing a frequency-selective MIMO channel into a set of parallel frequency-flat MIMO channels, OFDM diminishes the complexity of the MIMO receiver. [3].Though, MIMO-OFDM encounters crisis of elevated crest to normal power ratio to outcome in non-linear deformation of the passed on motion at lifted power enhancer [4]. The transmitter has a high PAPR in case of normal power which is basically reduced, with reference to a steady immersion control. In these cutting edge business remote structures, the PAPR issue is huger in uplink [5] in light of the fact that this is the limiting association to the extent extension and choice [6], portable incurable is compelled in sequence control, the adequacy of the control enhancer is basic.

Various strategies have been proposed for lessening in OFDM frameworks of PAPR for example cutting [7] [8], fractional transmit successions (PTS) [8] [9], particular mapping (SLM) [8] [10], tone reservation, and so on. Among all these PAPR decrease techniques, cutting is by all accounts the least difficult plan. Be that as it may, it experiences in-band and out-of-band bending that embarrasses the execution of strategy. The in turn cube is separated into displace sub-obstructs in the PTS approach along these lines the sub-bearers in each sub-piece are weighted by stage factor pivots. The stage pivots are picked so that the PAPR is limited [11], [12].Selected Mapping (SLM) strategy is the most skilled diminishment technique to decrease (PAPR) of Orthogonal Frequency Division Multiplexing (OFDM) system[13] without flag twisting.

\section{PROPOSED PAPR REDUCTION TECHNIQUE}

For the diminishment of this PAPR a considerable measure of strategies has been introduced beneath. Here we have proposed two PAPR diminishment procedures in parallel way. They are

$>$ Partial Transmit Sequence (PTS)

$>$ Selected Mapping (SLM)

\subsection{Partial transmit sequence (PTS) Method}

In MIMO-OFDM signals, PTS procedure is for the most part used to lessen the PAPR decrease. This technique at first parts the recurrence vector into few squares. Before applying the stage changes in MIMO-OFDM signals the above recurrence division method is finished.
Revised Manuscript Received on August 14, 2019.

K.Aruna Kumari, Sr.Asst.Professor, Department of Electronics \& Communication Engineering, PVP Siddhartha Institute of Technology, Kanuru, Vijayawada, AP, India. (E-mail: gudipudiak@gmail.com)

Dr.K.Sri Rama Krishna, Professor\& HOD, Department of Electronics \& Communication Engineering, VR Siddhartha Engineering College, Kanuru, Vijayawada, AP, India. 


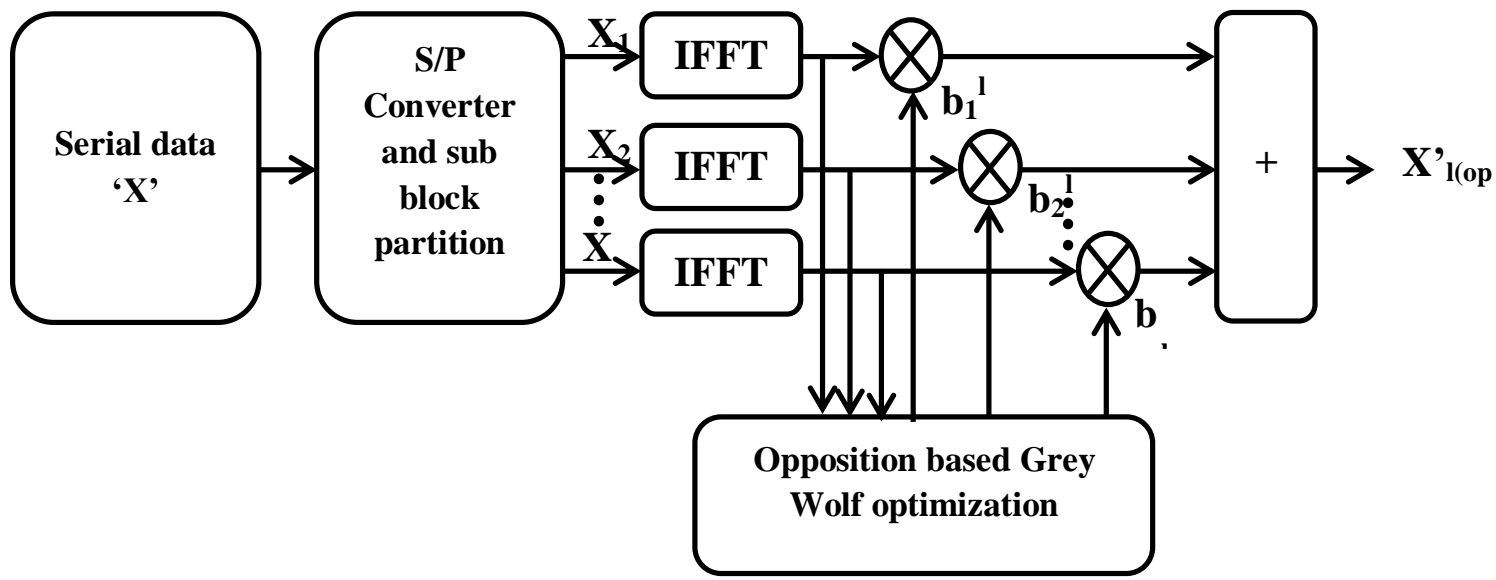

Fig 1: Block diagram of PTS

The practical layout of the PTS method is spoken to in the above figure. 1. Square parcel is used to disconnect the data frequencies into different pieces in PTS system. With the help of IFFT each square is taken care of. At last, the fitting stage vector is browsed the gathering of stage vectors. The enhancement of b square speaks to the duties of picking the ideal stage vectors.

\subsection{Selected Mapping (SLM) Method}

From the first information obstruct various option OFDM signals is made at first and after that transmit the OFDM flag having minimum PAPR is the major idea of this framework. This is a compelling and twisting less strategy used for the PAPR reducing in OFDM.

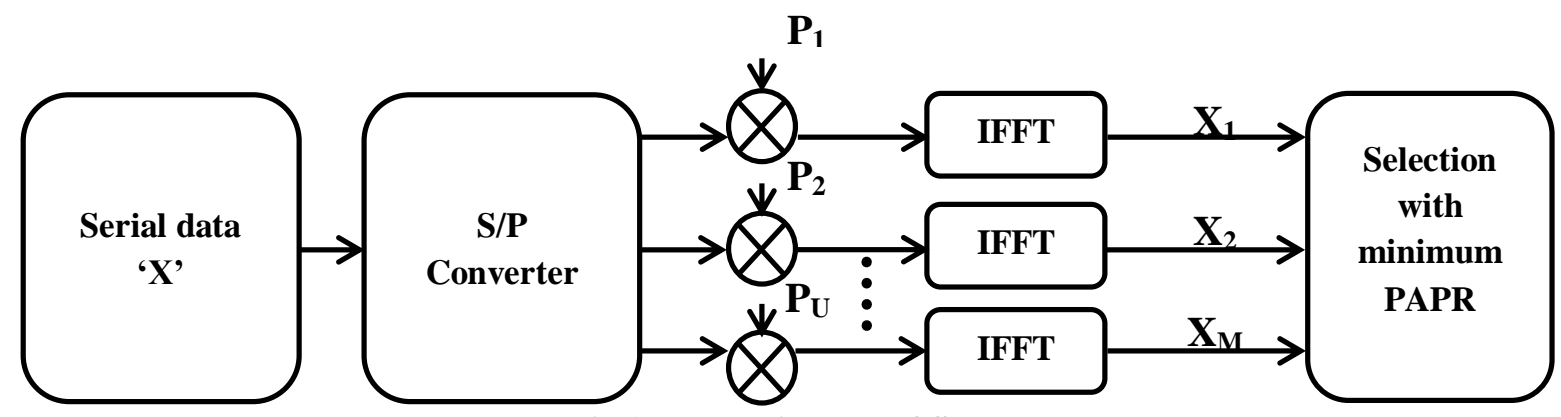

Fig 2: Block diagram of SLM

The figure 2 offers depiction about the transmitter side of the SLM framework. This chose OFDM motion at transmitter side must be seen at the collector. So the collector must have the data about the ideal stage vector that has been increased to create that chose OFDM flag.

\section{RESULT AND DISCUSSIONS}

The PAPR Reduction using PTS have been clubbed to attain better performance. The anticipated algorithm is executed with MATLAB software and the experimentation is carried out using a system of have 4 GB RAM and $2.10 \mathrm{GHz}$ Intel i-3 processor. This area demonstrates applicable implications of presented technique. Here, we have compared our presented system OGWO with GWO and $\mathrm{AABC}$ techniques.

\section{PERFORMANCE ANALYSIS}

In this section, the performance assessment for PAPR reduction using OGWO algorithm by means of PTS and SLM techniques and the existing algorithms like GWO and $\mathrm{AABC}$ is illustrated. The Bit Error Rate (BER), Symbol Error Rate (SER) and PAPR vs. CCDF values of proposed and existing methods are depicted as below in tables. From the comparison table 1, it shows the Bit Error Rate for both the proposed and existing methodologies by evaluated with different number of iterations. For the iteration number 100, the BER of

OGWO is 0.42 , GWO is 0.53 and for $\mathrm{AABC}$ is 0.61375 . Likewise, for the iteration 200, OGWO acquires 0.388125 , GWO achieves 0.495625 and 0.434375 is for AABC method. Thus the error acquired is minimum for our proposed method than the existing methods. It is clear that the error achieved is less for the proposed methodologies.

Table 1: Comparison of proposed and existing methodologies for BER calculation

\begin{tabular}{|c|c|c|c|}
\hline \multirow{2}{*}{ Iteration } & \multicolumn{3}{|c|}{ BER } \\
\cline { 2 - 4 } & OGWO & GWO & AABC \\
\hline $\mathbf{1 0 0}$ & 0.42 & 0.53 & 0.61375 \\
\hline $\mathbf{2 0 0}$ & 0.388125 & 0.495625 & 0.434375 \\
\hline $\mathbf{3 0 0}$ & 0.35875 & 0.46375 & 0.415938 \\
\hline $\mathbf{4 0 0}$ & 0.334375 & 0.43375 & 0.402188 \\
\hline $\mathbf{5 0 0}$ & 0.3 & 0.40625 & 0.39 \\
\hline $\mathbf{6 0 0}$ & 0.17625 & 0.275 & 0.380313 \\
\hline $\mathbf{7 0 0}$ & 0.147813 & 0.245 & 0.370625 \\
\hline $\mathbf{8 0 0}$ & 0.129063 & 0.229063 & 0.360625 \\
\hline $\mathbf{9 0 0}$ & 0.111875 & 0.212813 & 0.347188 \\
\hline $\mathbf{1 0 0 0}$ & 0.1 & 0.155313 & 0.289688 \\
\hline
\end{tabular}

Table 2: Comparison of proposed and existing

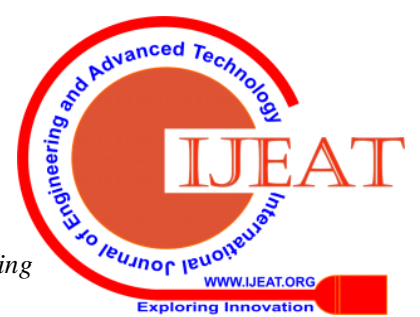


methodologies for SER calculation

\begin{tabular}{|c|c|c|c|}
\hline \multirow{2}{*}{$\begin{array}{c}\text { Iterati } \\
\text { on }\end{array}$} & \multicolumn{3}{|c|}{ SER } \\
\cline { 2 - 4 } $\mathbf{1 0 0}$ & OGWO & GWO & AABC \\
\hline $\mathbf{2 0 0}$ & 0.539375 & 0.541875 & 0.574375 \\
\hline $\mathbf{3 0 0}$ & 0.40875 & 0.515 & 0.555 \\
\hline $\mathbf{4 0 0}$ & 0.47375 & 0.495 & 0.54125 \\
\hline $\mathbf{5 0 0}$ & 0.458125 & 0.476875 & 0.53125 \\
\hline $\mathbf{6 0 0}$ & 0.444375 & 0.46 & 0.52 \\
\hline $\mathbf{7 0 0}$ & 0.43 & 0.445625 & 0.510625 \\
\hline $\mathbf{8 0 0}$ & 0.41375 & 0.4325 & 0.499375 \\
\hline $\mathbf{9 0 0}$ & 0.3925 & 0.3925 & 0.484375 \\
\hline $\mathbf{1 0 0 0}$ & 0.280625 & 0.280625 & 0.465 \\
\hline
\end{tabular}

The table2 shows that the comparison between SER with different iteration and evaluated the error rate for both the proposed and existing methodologies. For the iteration level 100, the proposed method OGWO acquires less error rate 0.539375 comparing to the existing techniques like GWO and $\mathrm{AABC}$ with 0.541875 and 0.574375 error rates respectively.

Table 3: Comparison of CCDF vs. PAPR for proposed and existing methodologies

\begin{tabular}{|c|c|c|c|}
\hline \multirow{2}{*}{ CCDF } & \multicolumn{3}{|c|}{ PAPR } \\
\cline { 2 - 4 } & OGWO & GWO & AABC \\
\hline $\mathbf{2}$ & 0.001 & 0 & 0 \\
\hline $\mathbf{2 . 5}$ & 0.03 & 0.025 & 0.01 \\
\hline $\mathbf{3}$ & 0.204 & 0.171 & 0.107 \\
\hline $\mathbf{3 . 5}$ & 0.54 & 0.478 & 0.343 \\
\hline $\mathbf{4}$ & 0.817 & 0.758 & 0.603 \\
\hline $\mathbf{4 . 5}$ & 0.942 & 0.928 & 0.816 \\
\hline $\mathbf{5}$ & 0.988 & 0.982 & 0.931 \\
\hline $\mathbf{5 . 5}$ & 0.996 & 0.994 & 0.979 \\
\hline $\mathbf{6}$ & 0.997 & 0.995 & 0.992 \\
\hline $\mathbf{6 . 5}$ & 0.999 & 0.994 & 0.993 \\
\hline $\mathbf{7}$ & 0.1 & 0.999 & 0.996 \\
\hline
\end{tabular}

Likewise for the iteration 200 the proposed method OGWO obtains the minimum error rate 0.50875 whereas GWO attains 0.515 and $\mathrm{AABC}$ with 0.555 error rate. Thus the result obtained for SER with all other iteration have achieved minimum error rate for proposed methodology OGWO as compared to the existing technologies like GWO and AABC.

Table 2 gives the resultant data obtained for PAPR vs. CCDF for both the proposed and existing methodologies. By calculating the PAPR vs. CCDF, proposed method OGWO obtains the maximum value when compared with other existing technologies. The bit error ratio is shown in figure 2.

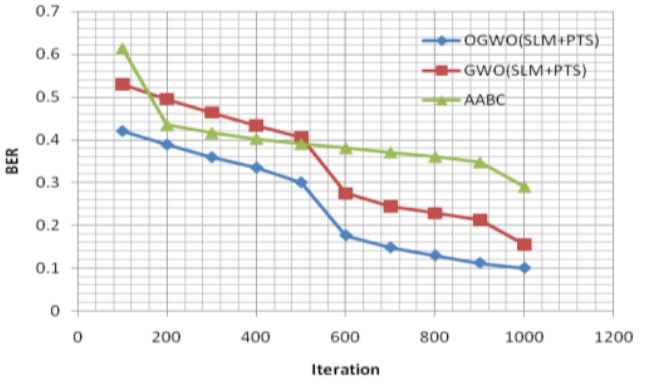

Fig 2: BER for proposed and existing methodologies

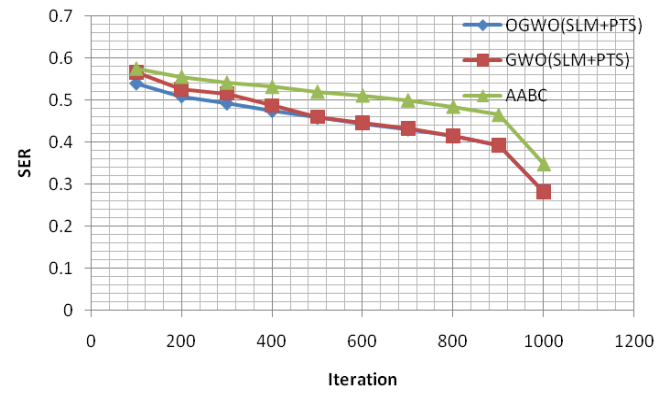

Fig 3: SER for proposed and existing methodologies

It container be pragmatic from over form that, mark error ratio for the anticipated system is declining much nearer than existing techniques. besides anticipated process showed superior results than presented method.

The proposed PAPR vs. CCDF graph for the projected and the offered technique is specified in figure 3 below.

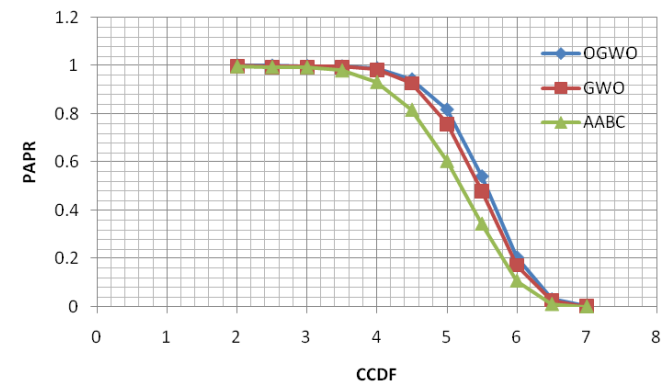

Fig 4: Comparison of PAPR vs. CCDF for proposed and existing methodologies

It canister be noted from beyond figure that PAPR drop takes put in each system but for the anticipated system PAPR takes place in short stage of time. besides, the PAPR has been reduced whereas; it is more in the existing technique. The major intention of the work was to achieve fewer PAPR as much as probable, which proves the competence of the system.

\section{CONCLUSION}

In this paper, we have anticipated oddity based Oppositional Gray Wolf Optimizer (OGWO) calculation utilizing SLM and PTS in parallel frame to decrease PAPR in MIMO-OFDM framework. For remote correspondences, OFDM is an exceptionally alluring strategy because of its range productivity and channel 

INPUT MULTI OUTPUT

vigor. The transmitted flag shows a high PAPR when the information groupings are corresponded is the one of the significant disadvantages in MIMO-OFDM frameworks. In the present work, two divergent PAPR diminishment strategies, i.e., SLM and PTS have been executed on the MIMO-OFDM conspire and the PAPR lessening parameter has been dissected. The result demonstrates that both the SLM plan and PTS plot are more compelling to diminish PAPR in MIMO-OFDM frameworks. With the reproduction ponders, it has been demonstrated that the proposed calculation lessens PAPR prevalent than the current frameworks.

\section{REFERENCES}

1. ZID Sound, BOUALLEGUE Rodham," Low-Complexity PAPR Reduction Schemes Using SLM and PTS Approaches for Interleaved OFDMA" IEEE Communications Surveys \& Tutorials, Volume: 15, Issue: 4, 2013

2. Hogweed Yang, Alcatel Shanghai Bell," A Road to Future Broadband Wireless Access: MIMO-OFDM-Based Air Interface",IEEE Communications Magazine, Volume: 43, Issue: $1, .2005$

3. Francisco Sandoval , GwenaelPoitau , François Gagnon," Hybrid Peak-to-Average Power Ratio Reduction Techniques: Review and Performance Comparison" IEEE Access ,Volume: 5,2017

4. Sadhana Singh, Arvind Kumar," Performance Analysis of Adaptive Clipping Technique for Reduction of PAPR in Alamouti Coded MIMO-OFDM Systems", International Conference On Advances In Computing \& Communications, ICACC 2016

5. K. Anoh, C. Tanriover, and B. Adebisi, "On the optimization of iterative clipping and filtering for PAPR reduction in OFDM systems," IEEE Access, vol. 5, pp. 12004-12013, 2017,

6. F. Khan, LTE for 4G Mobile Broadband: Air Interface Technologies and Performance. Cambridge, U.K.: Cambridge Univ. Press, ch. 5, pp. 88-108.,2009

7. J. Armstrong. Peak-to-average power reduction for OFDM by repeated clipping and frequency domain filtering. IEE Electronic Lett 2002; 38: 246-247

8. Y. Rahmatallah, S. Mohan. Peak-to-average power ratio reduction in OFDM systems: A survey and taxonomy. IEEE communications surveys \& tutorials, vol. 15, no. 4, pp.1567-1592, fourth quarter, 2013

9. J. Hou, J. Ge, J. Li. Peak-to-average power ratio reduction of OFDM signals using PTS scheme with low computational complexity. IEEE Trans Broadcast; pp: 143-148, 2011

10. R. W. Bauml, R. F. H. Fisher, J. B. Huber. Reducing the peak-to-average power ratio of multicarrier modulation by selected mapping. IEE Electronic Lett, Pp2056-2057,1996

11. O. Kwon and Y. Ha, Multi-carrier PAP reduction method using suboptimal PTS with threshold, IEEE Transactions on broadcasting, Vol. 49, No. 2, 2003

12. S. H. Han and J. H. Lee, An Overview of Peak-To-Average Power Ratio Reduction Techniques for Multicarrier Transmission, IEEE Wireless Communications, April 2005

13. SnikdhoSworovHaque, Md. MunjureMowla , Md. MehediHasan , ShaumendraKumer Bain," An Algorithm for P APR Reduction by SLM Technique in OFDM with Hadamard Matrix Row Factor", Electrical Engineering and Information Communication Technology (ICEEICT), 2015

14. M.V.R. Vittal, K. Rama Naidu," A novel reduced complexity optimized PTS technique for PAPR reduction in wireless OFDM systems",Egyptian Informatics Journal, Volume 18, Issue 2, Pages 123-131, 2017
15. M.V.R. Vittal, K. Rama Naidu," A Novel Parallel Artificial Bee Colony Algorithm and its PAPR Reduction Performance Using SLM Scheme in OFDM and MIMOOFDM Systems" IEEE Communications Letters, Volume: 19, Issue: 10, Oct. 2015

16. SimingPeng, Aijunliu, Kewang, Xiaohuliang," PAPR Reduction of Multicarrier Faster-Than-Nyquist Signals With Partial Transmit Sequence",IEEE Access, Volume: 5,2017

17. Jun Hou, Xiangmo Zhao, Fengkui Gong, FeiHui, and JianhuaGe," PAPR and PICR Reduction of OFDM Signals with Clipping Noise-based Tone Injection Scheme", IEEE Transactions on Vehicular Technology, Volume: 66, Issue: 1,2017

18. Sen-Hung Wang, Kuan-Chou Lee, Chih-Peng Li," A LowComplexity Architecture for PAPR Reduction in OFDM Systems with Near-Optimal Performance", IEEE Transactions on Vehicular Technology, Volume: 65, Issue: 1,2016

19. Hyun-SeungJoo, Kee-Hoon Kim, Jong-Seon No, DongJoon Shin," New PTS Schemes for PAPR Reduction of OFDM Signals Without Side Information" IEEE Transactions on Broadcasting, Volume: 63, Issue: 3, 2017

20. D. Kumutha, N. Amutha Prabha," Hybrid STBC-PTS with enhanced artificial bee colony algorithm for PAPR reduction in MIMO-OFDM system",Journal of Ambient Intelligence and Humanized Computing, pp 1-17,2017 